\title{
¿Ejecutores, implementadores o agentes curriculares?
}

\section{Perfiles docentes en relación al curriculum ${ }^{i}$}

Executors, implementers or curricular agents?

Teacher profiles in relation to the curriculum

Executores, implementadores ou agentes de currículo?

Perfis de professores em relação ao currículo

\section{DOI: https://doi.org/10.18861/cied.2021.12.2.3048}

\section{Roxana Di Pizzo Mateus}

Consejo de Educación Secundaria, Administración Nacional de Educación Pública

Uruguay

roxanadipizzo@gmail.com

ORCID: 0000-0001-7939-7538

\section{Claudia Cabrera Borges}

Consejo de Formación en Educación, Administración Nacional de Educación Pública

Instituto de Educación, Universidad ORT Uruguay

Uruguay

ORCID: 0000-0002-1419-679

Recibido: 08/12/20

Aprobado: 10/03/21

Cómo citar: Di Pizzo Mateus, R. \& Cabrera Borges, C. (2021). ¿Ejecutores, implementadores o agentes curriculares? Perfiles docentes en relación al curriculum. Cuadernos de Investigación Educativa, 12(2). https://doi.org/10.18861/cied.2021.12.2.3048

\section{Resumen}

El presente trabajo recoge algunos de los hallazgos de la investigación realizada en el marco del Master en Educación de la Universidad ORT Uruguay, en la cual se estudió la relación docente-curriculum. Se propuso como problema a investiga el modo en que los profesores de la asignatura Educacion Social y Civica actuan como mediadores de un curriculum en la idntifcación de perfies docentes a partir de las decisiones curriculos abjestas por los profesores estudiados Se lapó un enfoque culitativo dentro de la modalidad interactiva fenomén de recopilación de información fue la entrevista semiestructurada aplicada a una muestra teórica de diez profesores de Educación Socialy Civica (ubicados en tres etapas de la carrera docente) de liceos públicos de Montevidez pro un Inspector de la Asignatura del Consejo do Educación Secundaria de Uruguay Se complementó con el análisis textual cunlitativo de la Asumentos sobon once planes anio $y$ cinco replanicaciones de los docentes seleccionados en la muestra. Entre los profesores participantes del estudio los resultades permitieron identificar mayormente los perfles ejecutor implementalor del curiculum No se identificaron docentes con un perfi netamente de a nte curicular La investigación puso de manifesto que es en el trabajo colaborativo y la incorporación de tecnologies digitales donde los profesores más se distancian del perfit agente curricular. Un hallazgo a destacar es la profundización del perfil ejecutor en instancias

Palabras clave: docente, curriculum, enseñanza secundaria, ciencias sociales, planificación.

\section{Abstract}

This report includes some of the findings of a research carried out in the framework of the Master's Degree in Education at ORT Unins issty of Uruguay, where the elationsh between in which secondary educotion teachers of the subject Social and Civic Education act as mediators of a curriculum conceived within a curricular system The article is focused on one of the objectives of the research consisting in identifying the teachers' profiles based on the curricular decisions made by those teachers that participated in the study. A qualitative approach was used within the hermeneutic phenomenological interactive modality. The main technique for information gathering was the semi-structured interview applied to theoretical sampling of 10 teachers of Social and Civic Education from state high school in Montevideo city lapplied through three different stages of their teaching career) and one teachers supervisor for such specific subject appointed by the secondary Education eleven annual plans and five re-plasning documents written by teachers selected in the sample As regards the teachers participating in the study the results allowed to identify mostly the executor and implementer profiles. No teachers with a profile of mere curriculer agents were detected. The research evidenced that it is in the collaborative work and with the incorporation of digital technologies where teachers take distance from the curricularagent profile. A finding to be pointed out is the deepening of the executor profile in collective actions such as teachers' meetings and coordination activities

Keywords: teacher, curriculum, secondary school teaching, social sciences, planning

\section{Resumo}

A presente análise contém alquns achados da pesquisa realizada no âmbito Mestrado em Educação na Universidade ORT Uruguai e na qual foi examinada a relação professorcurriculo. O objeto a pesquisar foi analisar se os professores que ministram os cursos da disciplina Organização Social e Politica atuam como mediadores de um curricuto pesquisa: identificar os perfis de professores com base nas decisoes curriculares tomadas por eles. Uma abordagem qualitativa fol usada dentro do modo interativo fenomenológico hermeneutico. A principaltecnica de coleta de informaçoes for a entrevista semiestruturada com uma amostra teórica de dez professores de Organização Social e Politica no ensino do Conselho de Ensino Secundário do Uruguai Foi complementado com uma análise textual qualitativa de documentos sobre onze planos anuais e cinco replanejamentos desenvolvidos pelos professores selecionados na amostra. Os resultados permitiram dentificar, principalmente, dentre os professores participantes da pesquisa, os perfis de execucão e implementacão do curriculo. Não foram identificados professores com um perfil de agente claramente curricular. A pesquisa mostrou que e no trabalho colaborativo e na Incorporaçaa de tecnologilas digitalis onde os professores se distanciam mais do perfil de agente curricular. Um achado a salientar e o aprofundamento do perfil de execução em coletivas como reuniões e coordenações de professores

Palavras-chave: professor, curriculo, ensino médio, ciências sociais, planejamento 


\section{Introducción}

El presente artículo se basa en una investigación realizada en el marco del Master en Educación de la Universidad ORT Uruguay, en la que se indagó el papel del profesor en el curriculum y, especificamente, de los profesores de Educación Social y Cívica. El propósito principal es compartir algunos hallazgos vinculados a uno de sus objetivos especificos: la identificación de perfiles docentes a partir de las decisiones curriculares adoptadas por los profesores estudiados.

El profesor y sus funciones, así como su incidencia en los procesos curriculares son cuestiones insoslayables en cualquier reflexión sobre el curriculum. Según González (1986), el reconocimiento de la naturaleza práctica y contextual del curriculum obliga a prestar atención al profesor como sujeto activo, con capacidad para tomar decisiones para la realización de las prácticas curriculares. Desde un enfoque de desarrollo curricular (Zabalza, 2009) el curriculum es concebido como un espacio decisional en el que la escuela y los profesores desempeñan papeles curriculares.

Por su parte Opertti (2013) afirma que las reformas educativas en el mundo de hoy ponen más énfasis en los enfoques curriculares, en tanto el curriculum es visualizado como centro de los cambios educativos. A nivel interregional, las reformas más eficaces atribuyen autonomía y sostén al nivel local en un marco común y universal de distribución de responsabilidades, dentro de un proceso de adaptación del currículo al contexto local (Opertti, 2013, párr.16)

En esta visión los docentes son codesarrolladores de los currículos y agentes dinámicos del cambio. Sin embargo, el autor afirma que "la realidad indica que son aprehensivos, cuestionan las reformas y los cambios que implementan" (Opertti, 2013, párr.25). En respuesta a esta resistencia, parte de los debates actuales según Opertti (2013) se centran en encontrar la forma de apoyarse en los conocimientos y las experiencias docentes.

Como forma de contribuir a ese conocimiento y experiencias de los docentes se consideró relevante estudiar la relación entre el curriculum y el profesor y determinar qué docentes tenemos en relación al curriculum.

\section{Líneas teóricas}

A continuación, se exponen las principales líneas teóricas que guiaron el análisis de los datos.

\section{Concepción práctica del curriculum: procesual, sistémica}

Distintos autores (Stenhouse, 1998; Kemmis, 1998; Gimeno Sacristán, 1991; Grundy, 1998; De Alba, 1994) adoptan una perspectiva del curriculum como praxis.

En la concepción de Gimeno Sacristán (1991) el curriculum se configura y objetiva como un proyecto que es resultado de decisiones culturales, económicas, políticas y pedagógicas, pero su realización posterior tiene lugar en un contexto práctico dentro del cualjuegan prácticas diversas. "Como tal proyecto configura en gran medida la práctica pedagógica, pero es a su vez delimitado y limitado en sus significados concretos por esa misma práctica que existe previamente a cualquier proyecto curricular" (Gimeno Sacristán, 1991, p. 29). En realidad, el curriculum forma parte de múltiples tipos de prácticas que se desarrollan a distintos niveles. 
Este planteo del autor resultó particularmente interesante en tanto aborda el estudio del curriculum dentro de un sistema curricular donde se entrecruzan determinaciones y componentes muy diversos: pedagógicos, políticos, administrativos, de control, de innovación pedagógica, entre otros, lo cual permite un abordaje sistémico y procesual del curriculum.

En relación a dichas determinaciones cabe mencionar el estudio de Monarca y Fernández-González (2016) en el que se analizan los procesos relacionados con una innovación educativa en España. En este estudio se incorpora un elemento que se consideró en la investigación realizada: los inspectores y su función de control o supervisión de la tarea docente y del apego de sus documentos al curriculum prescripto. Los resultados evidencian que el papel desempeñado por los inspectores educativos representa, en muchas casos, un elemento obstaculizador de las innovaciones propuestas, principalmente por una excesiva vigilancia de los documentos de los centros.

Por último, desde este enfoque práctico o procesual, "(...) el curriculum es un objeto que se construye en el proceso de configuración, implantación, concreción y expresión de unas determinadas prácticas pedagógicas" (Gimeno Sacristán, 1991, p. 123). El curriculum involucra campos de acción diversos y múltiples agentes con distintas competencias y niveles a través de los cuales se va objetivando. A esos niveles de objetivación del significado del curriculum Gimeno Sacristán (1991) se refiere como: curriculum prescripto, curriculum presentado a los profesores, curriculum moldeado por los profesores, curriculum en acción, curriculum realizado y curriculum evaluado.

\section{El profesor como profesional activo: mediador del curriculum}

A través del curriculum moldeado por los profesores Gimeno Sacristán (1991) introduce la idea de mediación al análisis del desarrollo del curriculum. Esto significa concebir al profesor como un agente activo en el desarrollo curricular, un mediador decisivo entre el curriculum prescripto y los alumnos. Al mismo tiempo, sin embargo, el autor advierte que dadas las condiciones institucionales en las que ejercen su función docente, los profesores asumen un papel de gestores más que de diseñadores genuinos del curriculum.

Desde una óptica análoga a la de Gimeno Sacristán (1991), Marrero (2013) también refiere al profesor como mediador del curriculum. Entiende que el proceso de transformación del curriculum desde la esfera técnico-política a la prácticopedagógica requiere de la mediación del profesorado:

Ello implica concebir al profesor como un mediador decisivo entre el curriculum establecido y los alumnos. Supone, además, entender cómo realmente los espacios escolares son lugares de reconstrucción del conocimiento y de la práctica prefigurada por el curriculum, impuesto desde fuera de las instituciones educativas (p. 223).

Díaz Barriga (1995) introduce un aporte interesante para el estudio de la relación docente-curriculum al señalar la tensión que se plantea entre los procesos de institucionalidad de los planes y programas y los procesos académicos. En cuanto a los procesos institucionales existen dos concepciones acerca de los planes y programas: son la norma a cumplir o constituyen una orientación. En relación a los procesos académicos, serian el docente ejecutor/operario del programa dominado por una racionalidad burocrática y el docente intelectual reflexivo, que busca alternativas frente a las propuestas programáticas. 


\section{Desarrollo curricular y profesionalidad docente}

En la concepción de desarrollo curricular de Zabalza (2009), los centros educativos son creadores de curriculum y los profesores se convierten en profesionales activos en la construcción del mismo.

En la línea de Gimeno Sacristán (1991), Zabalza (2009) y Marrero (2013), Fernández (2004) concibe el desarrollo curricular como un espacio social y profesional de toma de decisiones sobre la escuela y la enseñanza. Según este autor, sin perjuicio de la legitimidad de las autoridades administrativas en el planteamiento de las propuestas curriculares, el curriculum se va formalizando en la práctica escolar a través de profesores y alumnos, por lo cual el desarrollo curricular puede analizarse como un ámbito de profesionalización del docente.

En este sentido, Day (en Marcelo, 1999) afirma que el desarrollo curricular es desarrollo profesional, "cuando contribuye a mejorar el conocimiento y la profesionalidad de los profesores, cuando a través de ello los profesores pueden plantear transformaciones y mejoras en el ámbito de su espacio de influencias: las escuelas, el curriculum, la enseñanza" (p. 161).

Figura 1. Sintesis de la perspectiva teórica a la que se afilia la investigación

\begin{tabular}{|c|c|c|}
\hline Escuela como insti & n que crea su curriculum & \\
\hline $\begin{array}{l}\text { Curriculum: } \\
\text { concepción } \\
\text { práctica procesual }\end{array}$ & $\begin{array}{l}\text { Profesor como } \\
\text { profesional } \\
\text { activo }\end{array}$ & \\
\hline & & n \\
\hline $\begin{array}{l}\text { Desarrollo curricula } \\
\text { desarrollo profesior }\end{array}$ & $\begin{array}{l}\text { 1o ámbito de } \\
\text { cente }\end{array}$ & $\begin{array}{l}\text { l } \\
\text { i } \\
d \\
\text { a } \\
d\end{array}$ \\
\hline
\end{tabular}

Fuente: Di Pizzo (2019)

De los enfoques teóricos desarrollados surge la asociación entre desarrollo curricular, desarrollo profesional y cambio o innovación curricular.

En tal sentido, Tejada (1998) realiza una caracterización de los docentes en cuanto a su papel en la innovación curricular (tabla 1):

a) El profesor ejecutor, no influye en la innovación, se limita a desarrollar en la práctica fidedignamente las prescripciones técnicas y administrativas;

b) El profesor implementador mediador de la innovación. No es un mero consumidor de proyectos de innovación, los adapta a las necesidades de su contexto de actuación; y

c) El profesor agente curricular que es un técnico capaz de innovar: "Participa del diseño, reinterpreta el curriculum en su contexto, toma decisiones en función de las necesidades de la práctica [...] Construye la innovación" (Tejada, 1998, p. 128) 
Tabla 1. Papel del profesor en los procesos de innovación

\begin{tabular}{|l|l|l|}
\hline \multicolumn{1}{|c|}{ EJECUTOR } & \multicolumn{1}{|c|}{ IMPLEMENTADOR } & \multicolumn{1}{c|}{ AGENTE CURRICULAR } \\
\hline $\begin{array}{l}\text { Desarrollar fidedignamente } \\
\text { un proyecto }\end{array}$ & $\begin{array}{l}\text { Implementador activo } \\
\text { del proyecto }\end{array}$ & $\begin{array}{l}\text { Sujeto que filtra y redefine } \\
\text { proyectos }\end{array}$ \\
\hline $\begin{array}{l}\text { Papel secundario: } \\
\text { - excluido del diseño }\end{array}$ & Papel secundario: \\
- instrumento de desarrollo & - excluido del diseño & Papel primario: \\
& - - participa en el diseño \\
\hline
\end{tabular}

Fuente: Elaboración propia a partir de Tejada (1998)

Por su parte, Fernández (2004) aporta una caracterización de los profesores desde un enfoque que integra el desarrollo y la innovación curricular (tabla 2).

Tabla 2. Perspectivas sobre el desarrollo curricular y el papel del profesorado

\begin{tabular}{|l|l|l|l|}
\hline Enfoques/ Aspectos & Fidelidad & Adaptación mutua & Reconstrucción \\
& $\begin{array}{l}\text { Adopción } \\
\text { (implementación fiel) }\end{array}$ & $\begin{array}{l}\text { Adaptación } \\
\text { (desarrollo) }\end{array}$ & $\begin{array}{l}\text { Reconstrucción } \\
\text { (práctica emergente) }\end{array}$ \\
\hline Curriculum & $\begin{array}{l}\text { Propuesta especifica a } \\
\text { aplicar en la práctica }\end{array}$ & $\begin{array}{l}\text { Algo a adaptar, } \\
\text { de acuerdo con } \\
\text { factores personales y } \\
\text { contextuales }\end{array}$ & $\begin{array}{l}\text { Prácticas y experiencias } \\
\text { energentes, construidas } \\
\text { el aula/centro }\end{array}$ \\
\hline $\begin{array}{l}\text { Papel del } \\
\text { profesorado }\end{array}$ & $\begin{array}{l}\text { Consumidor, técnico y } \\
\text { ejecutor de directrices } \\
\text { externas }\end{array}$ & $\begin{array}{l}\text { Sujeto activo } \\
\text { en la } \\
\text { construcción y } \\
\text { adaptación } \\
\text { del curriculum }\end{array}$ & $\begin{array}{l}\text { Investigador que indaga } \\
\text { de modo intencional y } \\
\text { sistemático }\end{array}$ \\
\hline
\end{tabular}

Fuente: elaboración propia en base a la reelaboración de las principales perspectivas de la innovación curricular y el papel del profesorado de Escudero (en Fernández, 2004). 


\section{Metodología}

\section{Enfoque y técnicas de recolección de información}

En la investigación se empleó un enfoque cualitativo dentro de la modalidad interactiva fenomenológica hermenéutica (Hernández, Fernández y Baptista, 2014).

La principal técnica de recopilación de información fue la entrevista semiestructurada a una muestra teórica de diez profesores de Educación Social y Cívica de liceos públicos de Montevideo y a un inspector de la asignatura del Consejo de Educación Secundaria de Uruguay (CES).

Las entrevistas se realizaron entre los meses de octubre y diciembre de 2018. Esta técnica se complementó con el análisis textual cualitativo de documentos (Corbetta, 2007) sobre once planes anuales y cinco replanificaciones de los docentes seleccionados en la muestra.

El análisis de los planes anuales y replanificaciones docentes se fundamentó en la consideración de que la propuesta didáctico-pedagógica que los profesores explicitan en dichos documentos aporta evidencia empírica sobre las decisiones que adoptan en relación al curriculum. Asimismo, permite identificar en base a estas decisiones, perfiles que den cuenta del papel que asumen los docentes frente al curriculum prescripto.

\section{Unidades de análisis y selección de la muestra}

Se delimitó el alcance de esta investigación a los docentes de Educación Socialy Cívica de liceos públicos de Montevideo en 2018.

La elección de liceos públicos se funda en el enfoque de desarrollo profesional docente y la perspectiva sistémica del curriculum (Gimeno Sacristán, 1991; Marrero, 2013: Zabalza, 2009; Fernández, 2004) que adoptó la investigación y que involucra una doble dimensión: institucional y pedagógica.

El enfoque seleccionado determinó que fueran particularmente relevantes para la investigación el conocimiento y experiencia que tuvieran estos profesores de la asignatura y de las condiciones institucionales en que se lleva a cabo la tarea docente.

De la información brindada por la Inspección de Educación Social y Civica del CES se supo que la forma en la que este designa a sus docentes, sumado a la realidad de la asignatura (en especial su carácter deficitario por haber más cantidad de docentes que horas disponibles para ser elegidas), determina que en las instituciones públicas se desempeñen, en su gran mayoría, docentes titulados.

Con la intención de contemplar las dimensiones aludidas y tomando en cuenta la información referida, se decidió estudiar a profesores de dicha asignatura que se desempeñaran en el sistema educativo público.

La decisión de acotar la población objeto de estudio al departamento de Montevideo obedeció principalmente a la disponibilidad de recursos. 
Como criterio de selección de la muestra se empleó un muestreo intencionado teórico de variación máxima. Para lograr una muestra de variación máxima en el sentido atribuido por Hernández Sampieri et al. (2014), o asegurar la heterogeneidad estructural del objeto de estudio (Mejía Navarrete, 2000) se contempló la siguiente diversidad: de etapas en el desarrollo profesional docente, de ciclo de enseñanza secundaria y geográfica.

En cuanto al primero de los niveles estructurales, para contemplar la perspectiva teórica referida y el enfoque de desarrollo profesional adoptado se consideró pertinente recurrir al estudio de Huberman (1990) sobre las etapas en la vida profesional de los docentes.

En tal sentido se seleccionó una muestra con profesores de tres etapas por considerarse las más significativas. Estas etapas serian: de 4 a 6 años, de 7 a 25 años y de más de 25 años de actuación. Huberman (1990) caracteriza estas etapas como: de estabilización, de diversificación, activismo y de búsqueda de una situación profesional estable y de preparación para la jubilación, respectivamente.

En la figura 2 se presentan las etapas seleccionadas con las principales caracteristicas identificadas por Huberman (1990).

Figura 2. Etapas o ciclos de la carrera

\begin{tabular}{l|l}
\hline $\begin{array}{l}\text { ESTABILIZACIÓN } \\
\text { (4 a } 6 \text { años) }\end{array}$ & $\begin{array}{l}\text { - Dominio de un repertorio pedagógico } \\
\text { - Capacidad de selección de estrategias metodológicas y recursos } \\
\text { en función de los estudiantes }\end{array}$ \\
\hline $\begin{array}{l}\text { DIVERSIFICACIÓNY } \\
\text { ACTIVISMO }\end{array}$ & - Preocupación por la mejora docente \\
(7 a 25 años) & - Experimentando nuevas prácticas \\
& $\begin{array}{l}\text { - Búsqueda de promoción profesional a través del desempeño } \\
\text { de funciones administrativas }\end{array}$ \\
\hline $\begin{array}{l}\text { BÚSQUEDA DE UNA } \\
\text { SITUACIÓN ESTABLE Y } \\
\text { PREPARACIÓN PARA LA } \\
\text { JUBILACIÓN } \\
\text { (más de 25 años) }\end{array}$ & - Replanteamiento, crisis profesional \\
\hline
\end{tabular}

Fuente: elaboración propia a partir de Huberman (1990)

Trasladando estas etapas a lo previsto por el Estatuto del Funcionario Docente de la Administración Nacional de Educación Pública respecto a la antigüedad funcional, esta decisión implicó seleccionar docentes efectivos que estuvieran atravesando el $2 .^{\circ}, 3 .^{\circ}, 4^{\circ}$ y el $7 .^{\circ}$ grado.

Los participantes fueron seleccionados entre los docentes de mayor puntaje que, a su vez, en 2018 tuvieran a su cargo al menos un grupo de la asignatura seleccionada en el subsistema y lo ejercieran efectivamente. Esta exigencia del desempeño efectivo se fundamentó por la relevancia al momento de la recolección de datos y experiencias actuales.

Con respecto al segundo y tercer nivel de heterogeneidad referidos, se procuró que participaran de la muestra docentes de primer y segundo ciclo de educación secundaria, de liceos de las tres zonas en las que el CES divide el departamento de Montevideo: centro, este y oeste. 
Por último, y teniendo en cuenta un criterio de predisposición (Mejía Navarrete, 2000) entre los profesores que respondian al rango de variación o heterogeneidad estructural del objeto se seleccionó a aquellos cuatro mejor ubicados en el Escalafón Docente que, a su vez, manifestaran disposición a participar en la investigación.

\section{Reclutamiento de los participantes}

Para reclutar a los participantes se empleó el Escalafón Docente del CES 2018 ya que en él se encuentran diferenciados por asignatura y ordenados por antigüedad y aptitud docente.

El procedimiento de reclutamiento de los participantes consistió en contactar, siguiendo el orden escalafonario (en cada uno de los tramos seleccionados), a los profesores efectivos que cumplieran con los criterios metodológicos establecidos.

Como primer paso se identificaron los liceos en los que los profesores tenian radicadas sus horas docentes. El segundo paso consistió en determinar en cada uno de estos centros los siguientes factores: el nivel de enseñanza que imparten (ciclo básico o bachillerato) y su localización de acuerdo a la zonificación del CES (centro, este y oeste). Por último, y siguiendo el orden de prelación escalafonaria, se contactó a los cuatro primeros docentes de la asignatura con los que se lograra cubrir los criterios referidos.

La muestra final se representa en la tabla 3

Tabla 3. Muestra

\begin{tabular}{|c|c|c|c|c|c|c|}
\hline Etapa & Grado & Ciclo & Zona & $\begin{array}{l}\text { Entrevista } \\
\text { Código }\end{array}$ & $\begin{array}{l}\text { Plan anual } \\
\text { Código }\end{array}$ & $\begin{array}{l}\text { Re-planificación } \\
\text { Código }\end{array}$ \\
\hline \multirow{4}{*}{$\begin{array}{l}\text { Estabilización } \\
\text { Huberman } \\
\text { (1990) }\end{array}$} & $2 .^{\circ}$ & básico & Este & UA8 & PUA8 & $\begin{array}{l}\text { El profesor no } \\
\text { replanificó }\end{array}$ \\
\hline & $2 .^{\circ}$ & bachillerato & Oeste & UA1 & PUA1 & $\begin{array}{l}\text { El profesor no } \\
\text { replanificó }\end{array}$ \\
\hline & $2^{\circ}$ & básico & Este & UA3 & PUA3 & RPUA3 \\
\hline & $2 .^{\circ}$ & básico & Oeste & UA11 & PUA 11 & $\begin{array}{l}\text { El profesor no } \\
\text { replanificó }\end{array}$ \\
\hline \multirow{3}{*}{$\begin{array}{l}\text { Diversificación } \\
\text { y activismo } \\
\text { Huberman } \\
\text { (1990) }\end{array}$} & $3 .^{\circ}$ & bachillerato & Este & UA7 & PUA7 & $\begin{array}{l}\text { El profesor no } \\
\text { replanificó }\end{array}$ \\
\hline & $30^{\circ}$ & bachillerato & Centro & P9 & $\begin{array}{l}\text { P1UA9 } \\
\text { P2UA9 }\end{array}$ & $\begin{array}{l}\text { El profesor no } \\
\text { replanificó }\end{array}$ \\
\hline & $4 .^{\circ}$ & bachillerato & Oeste & UA2 & PUA2 & $\begin{array}{l}\mathrm{RP} 1 \cup A_{2} \\
\mathrm{RP} 2 \cup A_{2}\end{array}$ \\
\hline \multirow{4}{*}{$\begin{array}{l}\text { Búsqueda de } \\
\text { una situación } \\
\text { profesional } \\
\text { estable } \\
\text { y preparación para } \\
\text { la jubilación } \\
\text { Huberman (1990) }\end{array}$} & $7 .^{\circ}$ & bachillerato & Centro & UA4 & PUA4 & $\begin{array}{l}\text { El profesor no } \\
\text { replanificó }\end{array}$ \\
\hline & $7^{\circ}$ & bachillerato & Centro & UA5 & \multicolumn{2}{|c|}{$\begin{array}{l}\text { Se elimina a solicitud del } \\
\text { participante }\end{array}$} \\
\hline & $7^{\circ}$ & bachillerato & Este & UA6 & PUA6 & $\begin{array}{l}\text { El profesor } \\
\text { no replanificó }\end{array}$ \\
\hline & $7^{\circ}$ & bachillerato & Centro & UA10 & PUA10 & RPUA10 \\
\hline
\end{tabular}

Fuente: elaboración propia 


\section{Categorías de análisis}

Para identificar en los profesores estudiados los perfiles docentes en relación al curriculum (según sus decisiones e intervenciones sobre el programa de la asignatura) se construyeron a priori tres grandes categorias teóricas adaptando la caracterización de Tejada (1998) sobre el papel del profesor en la innovación curricular.

Estas categorías son: profesor ejecutor (tabla 4), profesor implementador (tabla 5) y profesor agente curricular (tabla 6).

Tabla 4. Categorización. Profesor ejecutor

\begin{tabular}{|c|c|c|}
\hline CATEGORIAS & SUBCATEGORIAAS & \\
\hline \multirow{3}{*}{$\begin{array}{l}\text { Perfil del profesor } \\
\text { ejecutor (Tejada, 1998) }\end{array}$} & \multirow{3}{*}{$\begin{array}{l}\text { Cumplimiento de las } \\
\text { prescripciones normativas } \\
\text { y del cuerpo técnico y } \\
\text { administrativo (Tejada, 1998) }\end{array}$} & Seguimiento del programa \\
\hline & & $\begin{array}{l}\text { Cumplimiento de las pautas } \\
\text { de Inspección de Asignatura }\end{array}$ \\
\hline & & $\begin{array}{l}\text { Cumplimiento de los } \\
\text { requerimientos de } \\
\text { la Dirección Liceal e } \\
\text { institucionales: Salas } \\
\text { Docentes y Coordinaciones }\end{array}$ \\
\hline
\end{tabular}

Fuente: elaboración propia a partir de Tejada (1998)

De manera análoga a la categoría profesor ejecutor, se construyó para la categoría docente implementador siguiendo las caracteristicas asociadas al perfil por Tejada (1998), la categoria Capacidad de iniciativa y toma de decisiones (capacidad reflexiva y critica).

Siguiendo la misma lógica que en la categoría perfil ejecutor, se diseñaron las siguientes subcategorías (tabla 5): relacionadas con el seguimiento del programa y pautas de Inspección sobre el cumplimiento del mismo, y relacionadas con los requerimientos de la Dirección Liceal e institucionales.

Tabla 5. Categorización. Profesor implementador

\begin{tabular}{|c|c|c|c|}
\hline CATEGORÍAS & SUBCATEGORÍAS & & \\
\hline \multirow[t]{2}{*}{$\begin{array}{l}\text { Perfil del profesor } \\
\text { implementador } \\
\text { (Tejada, 1998) }\end{array}$} & \multirow[t]{2}{*}{$\begin{array}{l}\text { Capacidad de } \\
\text { iniciativa y toma de } \\
\text { decisiones: capacidad } \\
\text { reflexiva y critica } \\
\text { (Tejada, 1998) }\end{array}$} & $\begin{array}{l}\text { Seguimiento del } \\
\text { programa y pautas } \\
\text { de Inspección sobre } \\
\text { el cumplimiento del } \\
\text { mismo }\end{array}$ & $\begin{array}{l}\text { - Cambios en el orden o } \\
\text { secuencia del programa } \\
\text { - Selección de contenidos } \\
\text { programáticos } \\
\text { - Propuesta metodológica } \\
\text { propia } \\
\text { - Planificación en función del } \\
\text { diagnóstico de intereses y } \\
\text { necesidades de los alumnos }\end{array}$ \\
\hline & & $\begin{array}{l}\text { Cumplimiento } \\
\text { de los } \\
\text { requerimientos de } \\
\text { la Dirección Liceal e } \\
\text { institucionales }\end{array}$ & $\begin{array}{l}\text { - Planificación en función del } \\
\text { proyecto institucional } \\
\text { - Planificación en función } \\
\text { de acuerdos de sala de } \\
\text { asignatura y coordinaciones }\end{array}$ \\
\hline
\end{tabular}

Fuente: elaboración propia a partir de Tejada (1998) 
En la misma linea seguida para los perfiles ejecutor e implementador se construyeron para el docente agente curricular siguiendo los rasgos asociados a este perfil por Tejada (1998) las categorias: poder-autonomía para intervenir, flexibilidad, trabajo en equipo y presencia de otros medios (nuevas tecnologías). El diseño de estas categorías y subcategorías se muestra en la tabla 6.

Tabla 6. Categorización: Profesor agente curricular

\begin{tabular}{|l|l|l|}
\hline CATEGORÍAS & \multicolumn{2}{|l|}{ SUBCATEGORÍAS } \\
\hline \multirow{2}{*}{$\begin{array}{l}\text { Perfil del profesor } \\
\text { agente curricular } \\
\text { (Tejada, 1998) }\end{array}$} & Foder-autonomía para intervenir (Tejada, 1998) \\
\cline { 2 - 3 } & Frabajo en equipo (Tejada, 1998) & $\begin{array}{l}\text { Necesidad del cambio: cambio } \\
\text { de actitud y adquisición } \\
\text { de nuevas competencias } \\
\text { profesionales }\end{array}$ \\
\cline { 2 - 3 } & $\begin{array}{l}\text { Voluntad de } \\
\text { autoperfeccionamiento }\end{array}$ \\
\cline { 2 - 3 } & $\begin{array}{l}\text { Presencia de otros medios } \\
\text { (Tejada, 1998) }\end{array}$ & $\begin{array}{l}\text { Nuevas tecnologías } \\
\text { Planificación del empleo de } \\
\text { recursos tecnológicos }\end{array}$ \\
\hline
\end{tabular}

Fuente: elaboración propia a partir de Tejada (1998)

\section{Presentación y discusión de los resultados}

A continuación se presentan los resultados obtenidos que muestran los perfiles identificados en la población estudiada y la correspondiente caracterización de los mismos basada en evidencia empírica.

\section{Profesor ejecutor}

Para sistematizar el análisis se decidió agrupar las subcategorias que responden al cumplimiento de las prescripciones normativas del cuerpo técnico y administrativo en las siguientes:

- Las relacionadas con el seguimiento del programa,

- Las relacionadas con el cumplimiento de las pautas de Inspección de Asignatura,

- Las relacionadas con los requerimientos de la Dirección Liceal (Proyecto de Centro), y

- Las relativas a requerimientos institucionales (Salas docentes y Coordinaciones).

\section{Relacionadas con el seguimiento del programa}

De los docentes entrevistados, tres señalan que estructuran su plan anual en base a los objetivos del programa. Incluso, entre ellos, un profesor manifiesta plantearse en su planificación anual los mismos objetivos del programa oficial. 
Ningún docente manifiesta incorporar al plan anual la totalidad de los contenidos programáticos, lo cual supone una selección de contenidos. En relación al perfil docente implementador habrá que analizar si constituye una verdadera jerarquización tomando como criterio especialmente al contexto.

A pesar de lo declarado por los docentes en las entrevistas, en las planificaciones anuales no siempre se advierte esa selección de contenidos programáticos. En general los profesores siguen la secuencia de contenidos del programa oficial, básicamente en el orden de las unidades o módulos temáticos. Si realizan algún cambio o alteración en el orden es fundamentalmente por razones de tiempo.

En el caso de que realicen modificaciones, las mismas son mínimas y se efectúan en el correr del año lectivo a modo de ajustes al cronograma para poder llegar a cumplir con el programa.

Algunos docentes reconocen alterar el orden para vincular o establecer conexiones entre los temas. Otros manifiestan que deciden invertir el orden de alguna unidad por cuestiones puntuales, como el sistema electoral en un año de elecciones nacionales. En estos casos generalmente no hay una justificación por escrito en el plan anual. Y si bien puede adelantarse algún tema (incluso de otra unidad), el tema luego es retomado en el orden del programa, lo cual no hace más que confirmar que hay una tendencia general a seguir el orden o secuenciación del mismo.

Es de destacar que lo visualizado en las planificaciones anuales respecto al seguimiento de la secuencia y orden de contenidos del programa es coincidente con las declaraciones de los profesores. Si se realizan modificaciones, estas son mínimas y no se justifican en el plan.

\section{Relacionadas con las pautas de Inspección sobre el cumplimiento del programa}

Por un lado se infiere de las declaraciones de los profesores un convencimiento de que debe cumplirse con el programa lo más posible. Al mismo tiempo se infiere que cada docente tiene su propia idea acerca del cumplimiento del programa y que la misma no está supeditada siempre a las directivas de la Inspección o del sistema.

Este último aspecto es clave en la determinación del perfil docente ejecutor e implementador. Este permite desmarcar a los docentes que, como señala Tejada (1998), realizan un seguimiento del programa y una ejecución fiel de las pautas sobre su cumplimiento, de aquellos que son mediadores entre las prescripciones del curriculum y las demandas del contexto de actuación (liceo).

Por otro lado, desde la perspectiva de Díaz Barriga (1995) puede diferenciarse entre el docente ejecutor/operario de programa dominado por una racionalidad burocrática y el docente intelectual reflexivo, mediador reflexivo de las propuestas programáticas. Tomando en consideración la carrera profesional, entre aquellos que por las evidencias analizadas denotan un perfil más ejecutor se encuentran las UA1 y UA3 ubicadas en la primera de las etapas seleccionadas y la UA2 perteneciente a la segunda etapa.

En cuanto al cumplimiento total del programa como mandato, por un lado podría decirse que es una idea que aflora de forma recurrente. Y, por el otro, que no hay certeza entre los docentes sobre la vigencia de ese mandato que es relatado como un mandato histórico en la Inspección. 
Con el propósito de contrastar las declaraciones de los docentes sobre este punto se entrevistó a un inspector de la Asignatura. En cuanto al grado de cumplimiento del programa, sus declaraciones son contundentes y contradicen la recurrente idea de los docentes sobre la vigencia del mandato del cumplimiento total:

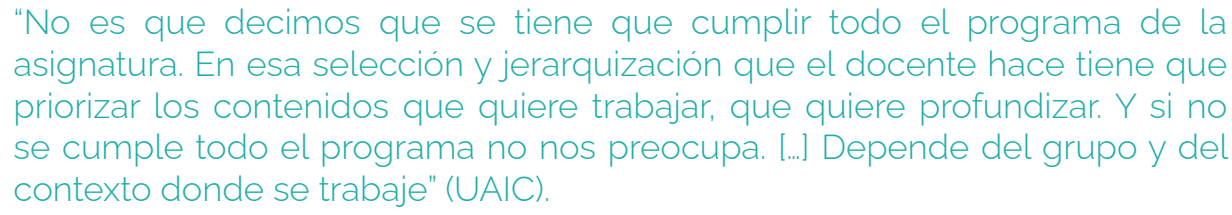

Respecto de la planificación de la metodologíay evaluación -e incluso la bibliografía incorporada al plan anual- se aprecian diferencias entre las manifestaciones de los docentes y la Inspección. Del testimonio de la Inspección se infiere un perfil docente en la asignatura netamente ejecutor:

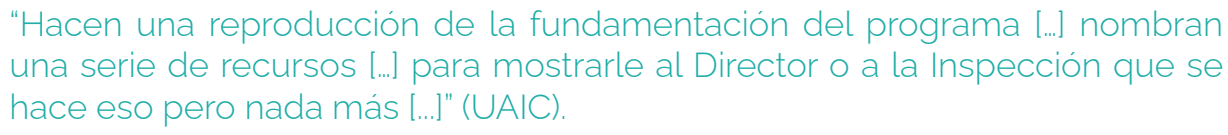

No obstante, del análisis de las entrevistas docentes surge que aquellos profesores que por sus dichos sobre la metodología y evaluación explicitada en sus planes muestran una propuesta propia (adaptando los recursos y estrategias o la evaluación, al estudiante, grupo, nivel o contexto) no son tan excepcionales. Si asimismo consideramos las planificaciones y replanificaciones de estos docentes, la cuestión no resulta tan clara ya que las mismas parecen confirmar la perspectiva del Inspector.

Tomando en consideración la investigación de Monarca y Fernández-González (2016), con el propósito de investigar la relación entre la función de la Inspección de supervisión de la tarea docente y del apego de sus documentos al curriculum prescripto con la eventual tendencia al perfil docente ejecutor, se formularon al inspector una serie de preguntas. Algunas de estas fueron:

- ¿Qué dice acerca de un docente su forma de planificar el curso?,

- Según su experiencia en la Inspección, ¿qué fortalezas y debilidades identifica en los profesores de su asignatura para planificar el curso?,

- ¿Existen diferencias entre la planificación de los mejores calificados en la visita y los que reciben menos puntaje?

Las preguntas formuladas apuntaron a contrastar los resultados de la referida investigación respecto a que la excesiva vigilancia de los documentos puede transformarse en un elemento obstaculizador de las innovaciones docentes.

Del análisis de las respuestas surge que el inspector entrevistado considera que el docente debe dirigir una buena enseñanza y su plan debe reflejarlo. Si bien, al decir de la Inspección, los profesores que mejor planifican obtienen mejor calificación en la visita, esta calificación no depende de un estricto apego al programa la las normas y/o pautas sobre su cumplimiento como mero formalismo) sino al grado de coherencia logrado entre los mismos.

En lo que interesa en este punto, no habría una excesiva vigilancia de la observancia de los documentos que lleve a los profesores a centrar sus esfuerzos en la realización de un documento lo mejor posible y, por ende favorecer un perfil docente ejecutor. 


\section{Relacionadas con los requerimientos de la Dirección Liceal}

Para indagar el cumplimiento de los requerimientos de la Dirección por parte de los docentes se les pregunta si en el liceo en que trabajan existe proyecto de centro y, en caso afirmativo, cómo se relaciona con la asignatura y si incorpora algún elemento del proyecto a su plan anual. Casi la mitad de los docentes manifiestan que en el liceo en que trabajan existe proyecto de centro.

Sus visiones y experiencias sobre estos proyectos son bien diferentes. La UA3 afirma que hay varios proyectos pero que ninguno se relaciona con la asignatura, razón por la cual no lo incorporó a su planificación.

Por su parte la UA10 sostiene que existe un proyecto de centro pero que el docente no lo conoce, aludiendo a una práctica que, según su opinión, también ocurre en otros centros.

En cambio, la UA11 afirma que en el liceo donde se desempeña como docente se trabajó mucho y seriamente en el proyecto de centro. En la triangulación con las planificaciones anuales y replanificaciones se pudo constatar que no hay mención, en ningún caso, al proyecto de centro.

\section{Relacionadas con requerimientos institucionales: salas docentes y coordinaciones}

La gran mayoria de los docentes entrevistados declaran incorporar a su plan anual o replanificaciones acuerdos de sala sobre contenidos programáticos y/o propuestas de evaluación (pruebas semestrales o exámenes).

Respecto de los contenidos, los profesores se ponen de acuerdo en la sala inicial sobre los temas a abordar durante el año. Y luego en la segunda parte del año estos acuerdos consisten en simples ajustes para lograr el cumplimiento del programa.

En cuanto a la evaluación, los acuerdos giran en torno a la estructura o formato de la prueba, y eventualmente, los contenidos que serán objeto de evaluación.

En cuanto a las coordinaciones con otras asignaturas, las expresiones de la UA3 son muy elocuentes en trasmitir los efectos prescriptivos que asumen muchas veces, y que llevan a los docentes a tomar decisiones forzadas para darles cumplimiento:

\footnotetext{
"Nos tocó trabajar en un mini proyecto con colegas de Historia y de Inglés [...] Entonces me dijeron: 'bueno, Cívica, entrá y hablános de las normas actuales y demás'. Y era como traído de los pelos y a mi me complicaba bastante" (UA3).
}

De las planificaciones anuales y replanificaciones analizadas no surge registro de estas instancias, a excepción del caso mencionado de la UA11 que se analiza en el perfil implementador. 


\section{Profesor implementador}

\section{Capacidad de iniciativa y toma de decisiones}

Para este perfil docente se considera pertinente presentar los resultados relacionados con el seguimiento del programa conjuntamente con las pautas de Inspección sobre su cumplimiento. De la misma forma, los requerimientos de la Dirección conjuntamente con los requerimientos institucionales.

\section{Relacionadas con el seguimiento del programa y pautas de Inspección}

En principio se tuvo en cuenta la toma de decisiones de los docentes respecto de la selección de contenidos y el orden o secuencia de los mismos en relación al programa oficial en tanto curriculum prescripto.

En el caso del perfil ejecutor la mirada estuvo puesta en el seguimiento del programa, las pautas de Inspección y los requerimientos institucionales y de la Dirección.

Para el perfil implementador, en cambio, la óptica se dirigió a la identificación de decisiones docentes que evidencien iniciativa propia y capacidad reflexiva y crítica respecto del curriculum prescripto.

Según Tejada (1998), el profesor implementador no es un simple ejecutor de proyectos: es un mediador. Siguiendo al autor, lo que caracteriza a este perfil docente es la capacidad de adecuar el proyecto de innovación (en este caso el programa) a su contexto de actuación.

En la investigación se adoptó una concepción amplia de contexto de actuación que incluye el aula, el nivel, el centro y el entorno comunitario.

Para el perfil implementador es necesario destacar dos casos: la UA4 y la UA1O que se encuentran transitando la última etapa de la carrera docente.

En ambos casos se aprecia la adecuación del programa al contexto: en el primer caso rompiendo con la linealidad de la secuencia prevista en el programa oficial y en el segundo articulándolo de acuerdo a los emergentes.

En ninguno de los casos sus planificaciones anuales y/o replanificaciones aportan evidencia que confirme una modificación de la secuencia de programa.

Respecto de la metodología y planificación de recursos y estrategias didácticas en el discurso de seis docentes se identificó una propuesta metodológica propia. Estos profesores trasmiten una preocupación constante por encontrar los recursos y estrategias más adecuados para el estudiante, grupo y/o contexto.

La planificación anual de la UA8, claramente la más extensa (19 páginas), es la más explícita en este punto. Incluye un cuadro en que describe los diversos estilos de aprendizaje (visual, auditivo y kinestésico), proponiendo para cada uno de ellos una metodología, estrategias y recursos didácticos acordes.

En el análisis del perfil ejecutor se identificó un grupo de docentes que tienen su propia idea del cumplimiento del programa, desligada de lo que se refirió como el mandato del cumplimiento total. Este grupo de profesores asocia el cumplimiento del programa con el cumplimiento de ciertos contenidos que prioriza según el contexto de actuación 
La UA7 lo expresa claramente citando un ejemplo en que un estudiante le pregunta si él también está "colgado de la luz"ii: "Trato de jerarquizar contenidos teniendo en cuenta los más pertinentes para el contexto en que están inmersos ellos [...] Porque el programa parte de la legalidad de las normas. Parte del supuesto de que todo el mundo las cumple".

Sin embargo, esta jerarquización a la que alude no pudo advertirse en su plan anual ya que los contenidos seleccionados no están explicitados. Dentro de los contenidos conceptuales se limita a transcribir los títulos de las unidades del programa oficial.

La percepción de este grupo de profesores sobre el grado de cumplimiento del programa estaría en sintonía con la pauta de Inspección analizada.

\section{Relacionadas con los requerimientos de la Dirección Liceal e institucionales}

La mitad de los profesores dicen incorporar a su plan anual elementos de la evaluación diagnóstica inicial, contextualizando su propuesta a las necesidades e intereses de los estudiantes. A excepción de la UA8, en ninguno de los casos surge de las planificaciones anuales que se haya tenido en cuenta y/o tomado decisiones sobre el curriculum en función de los resultados de la evaluación diagnóstica inicial.

Con respecto a la evaluación diagnóstica inicial, pero también a lo largo de la entrevista, la UAg marcó constantemente diferencias entre sus planificaciones según el turno: diurno o nocturno. Las diferencias señaladas no se explicarian, según el docente, por el tipo de población estudiantil sino por las características del plan de estudios. De hecho el docente ofreció para la investigación ambas planificaciones, las que fueron analizadas tras evaluar la pertinencia de contraponer planificaciones anuales de un mismo profesor dirigidas a poblaciones y planes de estudio diversos.

Respecto del proyecto de centro, en relación al perfil docente implementador interesan aquellos casos en que el profesor planifica integrando necesidades o temáticas surgidas del proyecto institucional, diseñando una propuesta contextualizada al centro.

Solo dos profesores refieren a su participación en proyectos de centro: la UA2 remite a su experiencia de años atrás en un liceo del interior del país y la UA11 a una experiencia actual en la que a partir del proyecto no solo trabajó con colegas de su asignatura sino también de otras asignaturas a través de las duplas. Las duplas constituyen una iniciativa del CES de coenseñanza en el aula, que propone una experiencia de trabajo interdisciplinario.

En cuanto a salas docentes y coordinaciones no hay indicios de temáticas, metodologías coordinadas o tipos o instrumentos de evaluación que respondan a las necesidades del contexto. Como se analizó en el perfil ejecutor, los acuerdos docentes están enfocados al cumplimiento de prescripciones administrativas tales como el seguimiento de los contenidos del programa y su evaluación. Se podría interpretar que responden en general más a una lógica de contralor que a una cultura profesional colaborativa. 


\section{Profesor agente curricular}

Cabe aclarar que el perfil agente curricular comparte los rasgos del perfil implementador en cuanto a la capacidad de iniciativa y toma de decisiones, a los que se suman poder-autonomía para intervenir, flexibilidad, trabajo en equipo y presencia de otras tecnologías.

\section{Poder-autonomía para intervenir}

Este rasgo se manifiesta en la entrevista con la UA4 a propósito de su forma de trabajar a partir de los intereses de los estudiantes: "En el programa no está Derecho laboral. Esto me lleva a pensar qué temas puedo retomar. Por ejemplo, hay una ley de acoso".

También de la entrevista con la UA7 se desprende este poder-autonomía: ".. Trabajar los clásicos pero desde la modernidad, más bien la posmodernidad". Tejada (1998) define este rasgo de la autonomía docente para intervenir, no tanto como una condición derivada del contexto sino derivada de la competencia del propio profesional.

En el caso de la UA4 el profesor demuestra ingenio para incorporar significativamente al programa temáticas que son del interés de los estudiantes, en este caso adultos que se desempeñan en el mercado laboral, sin que ello signifique alejarse de los contenidos del programa oficial. Por su parte la UA7 busca dar relevancia a los contenidos que pertenecen a un programa con más de diez años de vigencia.

Solo las declaraciones de la UA4 sobre la planificación en base a centros de interés de los alumnos quedan corroboradas en su planificación anual.

\section{Trabajo en equipo}

Lo analizado dentro del perfil implementador en relación a los requerimientos institucionales y de la Dirección Liceal aporta evidencias también para esta categoría. La clave para distinguir, en este aspecto, un perfil implementador de uno agente curricular está en los frutos del trabajo colaborativo en estas instancias colectivas. El perfil agente curricular supone un trabajo colaborativo fecundo entre los profesores, que se concrete en decisiones sobre el Curriculum.

Corresponde en este sentido volver a la UA11. En su plan anual expresa: "Este año, y como novedad, se suma a la planificación habitual la planificación en duplas" (PUA11). En otro punto incluye: "Proyecto en duplas [...] Visualizando la ciudadanía y Ciudadanía participativa desde el Arte" (PUA11). 


\section{Flexibilidad, necesidad del cambio, voluntad de autoperfeccionamiento}

Según Tejada (1998) el agente curricular es un docente "predispuesto a asumir el cambio como una constante de su actuación" (p. 130).

La mayoria de los profesores entrevistados manifiestan realizar cambios a su planificación anual. Dentro de estos docentes es posible distinguir entre aquellos que manifiestan hacer cambios menores a modo de ajustes para cumplir de mejor manera el programa, de aquellos que en sus declaraciones trasmiten sentir la necesidad de realizar cambios.

Estos últimos demuestran una predisposición al cambio y, por lo tanto, un perfil más flexible en el sentido atribuido por Tejada (1998).

Entre los profesores que denotan flexibilidad se encuentran la UAg, ubicada en la segunda de las etapas de la carrera docente seleccionadas y la UA8, ubicada en la primera etapa de la carrera docente.

Otro rasgo atribuido por Tejada (1998) al perfil agente curricular es la voluntad de perfeccionamiento.

Para indagar en este rasgo, a aquellos docentes que refirieron tener una actitud de cambio se les preguntó si esa necesidad los había conducido a seguir formándose. De las respuestas recogidas, tres conectan la necesidad del cambio a la formación. La UA11 es quien conecta de manera más explícita la necesidad del cambio con la formación. A lo largo de la entrevista es recurrente con la formación en dificultades de aprendizaje y lo alude como el foco principal de su interés profesional.

\section{Presencia de otros medios (tecnologías digitales)}

Del análisis de las entrevistas docentes surge que tres profesores declaran incluir en su planificación anual recursos digitales. Entre las herramientas digitales que dicen emplear las más recurrentes son: powerpoint, películas, cortometrajes y videos de youtube.

En la identificación del perfil docente agente curricular resulta significativa la presencia de un empleo planificado e innovador de esos recursos, entendiendo por innovador un uso que no implique la mera sustitución de recursos tradicionales como el pizarrón y/o para el caso de la asignatura educación social y cívica los textos sociológicos, la Constitución u otros textos normativos o, como señala Tejada (1998), la presencia de las nuevas tecnologias (en referencia a las tecnologias digitales), que permitan al profesor apartarse de la rutina y hacer propuestas que superen consignas tradicionales y memoristicas. La UA7 se expresa, precisamente, en ese sentido: "No si la usás simplemente para hacer lo mismo. Yo veo profesores que se dicen innovadores y lo único que hacen es traer un powerpoint y leerlo".

La UA8, profesor de la primera etapa docente, agrega: "Porque a los chiquilines no les gusta un video que les diga lo mismo que vos les estás diciendo. Como Educatina". 
La UA11, que también se encuentra transitando la primera de las etapas seleccionadas, reconoce estar haciendo sus primeros intentos de incorporar tecnología digital como forma de evaluación.

Estaría aqui gravitando una cuestión generacional, reconocida en las entrevistas por la mayoría de los profesores de la última etapa, que explicaría un vínculo más distante de estos docentes con la tecnología.

La planificación anual de la UA8 incluye un cuadro con un diseño muy atractivo por los colores e imágenes utilizados, en el que propone un variado repertorio de recursos didácticos según el estilo de aprendizaje del estudiante.

Tras el análisis de las entrevistas y de planificaciones y replanificaciones, en cuanto al trabajo colaborativo (en el sentido de incorporación significativa al plan anual y/o replanificaciones de acuerdos de sala, temáticas y/o metodologias coordinadas intra o interdisciplinariamente), solo se identificó el caso de la UA11 ubicada en la primera de las etapas de la carrera. Podría interpretarse que ese carácter excepcional se vincula más a la propuesta de duplas implementada en el centro educativo que a las características atribuibles a la etapa de la carrera docente en que se encuentra.

Respecto de la incorporación al plan anual de recursos tecnológicos con una auténtica intención innovadora, se encuentran los casos de la UA8 y la UA7, de la primera y segunda de las etapas de la carrera docente. Sin embargo, son también excepcionales dentro de las etapas a las que pertenecen.

En la primera de estas etapas, según Huberman (1990), el profesor comienza a desarrollar la capacidad de seleccionar metodologías y estrategias adecuadas a los intereses y necesidades de los estudiantes. Y en la segunda, dentro de los posibles recorridos profesionales refiere a aquellos que se centran en la mejora de sus prácticas diversificando estrategias metodológicas y experimentando prácticas innovadoras.

\section{Conclusiones}

El perfil ejecutor se manifiesta en los profesores estudiados en los siguientes aspectos: los objetivos propuestos, la rigidez en la planificación del tiempo, el seguimiento de los contenidos programáticos, la valoración del cumplimiento total del programa, el cumplimiento formal de las salas y coordinaciones docentes, la replanificación como ajuste al cronograma inicial o ajustes a la extensión o profundidad de los contenidos para dar cumplimiento total al programa, y en una planificación anual rutinaria.

Es principalmente en la metodología, evaluación y bibliografia donde la Inspección de la asignatura advierte características en los planes anuales de los profesores que se asocian al perfil ejecutor. El presente estudio no es del todo concluyente en este aspecto.

En cambio, sí se constató que gran parte de las decisiones que los docentes adoptan respecto del curriculum prescripto no están consignadas en el plan anual (o replanificaciones) y muchas incluso carecen de otro tipo de registro. Por tal motivo, un hallazgo de esta investigación es la existencia de planes mentales. 
A su vez, la evidencia recogida permite concluir que no existiría una excesiva vigilancia por parte de la Inspección de Asignatura de los documentos requeridos en sus visitas a docentes que favorezca un perfil ejecutor.

Asimismo, la investigación revela que la idea del cumplimiento total del programa oficial como mandato de la Inspección es recurrente entre los docentes y que existe diversidad de percepciones entre estos en cuanto a su vigencia.

Se considera que indagar sobre la vigencia de este mandato y, eventualmente, su poder de predisposición a un perfil docente ejecutor podria ser una futura línea de investigación.

Otra conclusión es que en las instancias institucionales colectivas, como salas y coordinaciones docentes, se profundiza una tendencia al perfil ejecutor de estos profesores. El estudio arroja que, lejos de existir una apropiación de los docentes de estos espacios, se reflejan los efectos del carácter obligatorio o prescriptivo de estas instancias que dejan en evidencia un tipo de docente cumplidor.

El perfil implementador se manifiesta en los docentes investigados, especialmente en la adecuación del plan anualal contexto deactuación a través de lajerarquización de contenidos del programa, la propuesta metodológica y la integración del proyecto de centro.

En consonancia con lo concluido respecto a la profundización del perfil ejecutor en instancias colectivas, no hay indicios de coordinación de temáticas, metodologias o de tipos o instrumentos de evaluación diferenciados que atiendan a las necesidades del contexto.

Por último, de la investigación no surgen docentes que se asocien completamente al perfil agente curricular. Por este motivo se prefiere hablar de profesores con potencial para asumir este perfil y referirse a ellos en términos de proximidad y distancia con dicho perfil.

La evidencia más clara de acercamiento al perfil agente curricular se manifiesta en planificaciones anuales en la que se intenta mejorar la relevancia del curriculum, concretamente estableciendo conexiones entre el programa oficial y la contemporaneidad a través de la incorporación de temáticas de actualidad que no están dentro de los contenidos programáticos (pero que se logran integrar) y que podrian incluirse dentro de las denominadas nuevas alfabetizaciones (Terigi, 2012).

La investigación pone de manifiesto que es en el trabajo colaborativo y la incorporación de tecnologías digitales donde los profesores más se distancian del perfil agente curricular.

Al respecto, no se halló evidencia de un trabajo colaborativo de los docentes en el que se realicen acuerdos que demuestren autonomía y que se concreten en decisiones sustantivas sobre el curriculum tales como incorporación de temáticas para mejorar su relevancia, nuevos enfoques intra o interdisciplinarios, metodología innovadora o propuestas de evaluación innovadoras y/o individualizadas.

Asimismo pudo advertirse que existen decisiones de los docentes respecto del curriculum prescripto que están determinadas o responden a requerimientos de un plan de estudios.

Se encontraron docentes que en un plan, o incluso a nivel de enseñanza reflejan un perfil más ejecutor, y en otro demuestran un perfil más implementador y hasta agente curricular. Por ello podría concluirse que el perfil docente (ejecutor, 
implementador o agente curricular) no está dado exclusivamente por las características personales y profesionales del docente como podría pensarse, o de la etapa en la carrera docente que esté transitando sino que intervienen también factores institucionales o del sistema.

Estos factores (no necesariamente vinculados a una excesiva prescripción, control o supervisión) pueden conducirlo a asumir, y a veces hasta a resignarse a asumir un perfil ejecutor. O como en otros casos, y por el contrario, puede desafiarlos a un perfil más implementador y hasta agente curricular.

En este sentido podría pensarse en una línea de investigación que indagara sobre esos modelos docentes que los profesores perciben como funcionales al sistema y en qué medida estos modelos promueven u obturan un perfil docente coherente con los cambios y la innovación educativa.

Asimismo la profundización del perfil ejecutor en instancias docentes colectivas deja planteada la pregunta de si ese perfil, más que responder a una lógica de contralor, estaría compensando una falta de competencia de los docentes de esta asignatura para el trabajo colaborativo.

En sintesis, la investigación arroja algo más de luz sobre esa red invisible entre determinaciones del curriculum y decisiones que lo configuran y que cita Marrero (2013), ya consignado, cuando habla de concebir al profesor como un mediador entre el programa y los alumnos.

\section{Notas:}

' Si bien la Real Academia Española define curriculo como plan de estudios y curriculum como la relación de los títulos y honores acerca de una persona, este segundo término también es ampliamente utilizado en el campo académico para referirse al plan de estudios.

ii La expresión "estar colgado de la luz" significa el robo de energía eléctrica -por medio de un cableado ilegal- a otro usuario que si paga por el servicio, práctica bastante generalizada en contextos socioeconómicos desfavorecidos.

Aprobación final del artículo: Editora responsable Mag. Verónica Zorrilla de San Martín Contribución de autoria: La concepción del trabajo científico fue realizada por Claudia Cabrera Borges y Roxana Di Pizzo Mateus. La recolección, interpretación y análisis de datos estuvo a cargo de Roxana Di Pizzo Mateus. La redacción del manuscrito fue realizada por Claudia Cabrera Borges y Roxana Di Pizzo Mateus. Ambas autoras revisaron y aprobaron el contenido final. 


\section{Referencias bibliográficas}

CORBETTA, P. (2007). Metodología y técnicas de investigación social. McGraw Hill. https://diversidadlocal.files.wordpress.com/2012/09/metodologc3ada-ytczagcnicas-de-investigacic3b3n-social-piergiorgio-corbetta.pdf

DE ALBA, A. (1994). Currículum: crisis, mito y perspectivas. Buenos Aires, Argentina: Miño y Dávila Editores. http://wwww.terras.edu.ar/biblioteca/1/CRRM_De_Alba_ Unidad_1.pdf

DÍAZ BARRIGA, A. (1995). Docente y programa. Lo institucional y lo didáctico. Aique Grupo Editor.

DI PIZZO, R. (2019). Profesores y desarrollo curricular. Decisiones, determinaciones y percepciones de los docentes de educación social y civica sobre el curriculum [Tesis de Maestría, Universidad ORT]. https://bibliotecas.ort.edu.uy/bibid/89845

FERNÁNDEZ, M. (2004). El desarrollo docente en los escenarios del curriculum y la organización. Revista de curriculum y formación del profesorado, 8(1), 1-20. https://Www.ugr.es/ recfpro/rev81COL3.pdf

GIMENO SACRISTÁN, J. (1991). El currículum: una reflexión sobre la práctica. Ediciones Morata.

GONZÁLEZ, M. (1986). El papel del profesor en los procesos de cambio. Revista interuniversitaria de didáctica, 4(4-5), 9-30. https://dialnet.unirioja.es/servlet/ articulo? codigo $=95627$

GRUNDY, S. (1998). Producto o praxis del curriculum. Ediciones Morata.

HERNÁNDEZ SAMPIERI, R., FERNÁNEZ COLLADO, C., \& BAPTISTA LUCIO, P. (2014). Metodología de la Investigación Social. MacGraw Hill. https://eva.fic.udelar. edu.uy/pluginfile.php/25828/mod_resource/content/1/Manual\%20de\%20 metodolog\%C3\%ADa\%20de\%20la\%20investigaci\%C3\%B3n\%20Sampieri.pdf

HUBERMAN, M. (1990). Las fases de la profesión docente. Ensayo de descripción y previsión. Curriculum, (2), 139-159. http://www.quadernsdigitals.net/index. php?accionMenu=hemeroteca.VisualizaArticulolU.visualiza\&articulo_id=2638

KEMMIS, S. (1998). El curriculum: más allá de la teoria de la reproducción. Ediciones Morata.

MARCELO, C. (1999). Formación del profesorado para el cambio educativo. Ediciones Universitarias de Barcelona.

MARRERO, J. (2013). El curriculum que es interpretado. ¿Qué enseñan los centros y los profesores y profesoras? En Gimeno Sacristán. (Comp.), Saberes e incertidumbres sobre el curriculum, 221-245. Ediciones Morata.

MEJíA NAVARRETE, J. (2000). El muestreo en la investigación cualitativa. Investigaciones Sociales, 4(5), 165-180. http://revistasinvestigacion.unmsm.edu. pe/index.php/sociales/article/view/6851

MONARCA, H, Y FERNÁNDEZ-GONZÁLEZ, N. (2016). El papel de la inspección educativa en los procesos de cambio. Cuadernos de Pesquisa, 46 (159), 212-233. http://dx.doi.org/10.1590/198053143374 
OPERTTI, R. (2013). Visión del curriculo y debates curriculares: una perspectiva interregional. https://es.calameo.com/read/0042763553edd458dog4b

STENHOUSE, L. (1998). Investigación y desarrollo del curriculum. Ediciones Morata.

TEJADA, J. (1998). Los agentes de innovación en los centros educativos. Profesores, directivos y asesores. Aljibe.

TERIGI, F. (2012). La cuestión curricular en la educación secundaria. En E. Tenti Fanfani (Coord.). La escolarización de los adolescentes: desafios culturales, pedagógicos y de política educativa, 55-75. IIPE-UNESCO.

ZABALZA, M. (2009). Diseño y desarrollo curricular. Narcea. 